\title{
Sismicidad antropogénica en los alrededores de algunas represas en Colombia
}

\author{
Mario Helberto Leal-Noriega ${ }^{{ }^{*}}$ (D) ; Carlos Alberto Vargas-Jiménez ${ }^{1}$ \\ Forma de citar: Leal-Noriega, M.H.; Vargas-Jiménez, C.A. (2020). Sismicidad antropogénica \\ en los alrededores de algunas represas en Colombia. Boletín de Geología, 42(3), 123-139. \\ https://doi.org/10.18273/revbol.v42n3-2020005
}

\begin{abstract}
Resumen
Esta investigación analiza si la sismicidad registrada en los alrededores de cinco embalses en Colombia, está relacionada con la sobrecarga y descarga ejercida por sus ciclos de llenado y vaciado, respectivamente. Las represas seleccionadas cuentan con diferentes rangos de volumen de almacenamiento de agua, por ser uno de los parámetros que más suelen influir en la sismicidad detonada por embalses. El período de estudio se delimitó entre los años 2000 y 2017, empleando como información de referencia los siguientes insumos: eventos sísmicos registrados por la Red Sismológica Nacional de Colombia, reportes diarios sobre el volumen de almacenamiento de los embalses, así como información geológico-estructural de las áreas de estudio a nivel regional generada por el Servicio Geológico Colombiano. Los análisis realizados para los embalses de Betania, Calima, Urrá y Salvajina, no permiten establecer de forma contundente una relación directa entre los sismos registrados y los cambios de volumen de estos embalses; sin embargo, los resultados obtenidos para la represa de Topocoro estiman posible que la construcción de esta estructura pueda estar incidiendo en la ocurrencia de una mayor cantidad de eventos sísmicos en sus alrededores.
\end{abstract}

Palabras clave: Sismicidad antropogénica; Sismicidad inducida; Embalses; Volumen almacenado; Sobrecarga.

\section{Anthropogenic seismicity in the surroundings of some reservoirs in Colombia}

\begin{abstract}
This research analyzes if the seismicity registered in the surroundings of five reservoirs in Colombia, is related to the overload and discharge exerted by its filling and emptying cycles, respectively. The selected dams have different water storage volume ranges, as they are one of the parameters that most often influence the seismicity detonated by reservoirs. The study period was delimited between 2000 and 2017, using the following inputs as reference information: seismic events registered by the National Seismological Network of Colombia, daily reports on the storage volume of the reservoirs, as well as geological-structural information of the study areas at the regional level generated by the Colombian Geological Service. The analyses carried out for the Betania, Calima, Urrá and Salvajina reservoirs do not allow to stablish a conclusive direct relationship between the recorded earthquakes and the volume changes of these reservoirs; however, the results obtained for the Topocoro dam estimate that the construction of this structure may be producing a greater number of seismic events in its surroundings.
\end{abstract}

Keywords: Anthropogenic seismicity; Induced seismicity; Reservoirs; Stored volume; Overload.

\footnotetext{
${ }^{1}$ Departamento de Geociencias, Universidad Nacional de Colombia, Bogotá, Colombia. (*) mhlealn@unal.edu.co; cavargasj@unal.edu.co
} 


\section{Introducción}

La literatura actual sobre la temática de sismicidad antropogénica, denominada a la actividad sísmica relacionada con la intervención humana en el medio ambiente (McGarr et al., 2002), destaca la relación encontrada hace varias décadas entre las grandes obras de ingeniería con propósitos de almacenamiento de agua y los sismos que se pueden presentar en las regiones más cercanas a estos proyectos; la cual se asocia principalmente con los cambios volumétricos que se presentan durante los procesos de llenado (Gupta, 2001) y vaciado de las represas en su etapa de funcionamiento.

Un ejemplo de esta relación destaca el sismo ocurrido el 10 de diciembre de 1967 en cercanía a la represa de Koyna (India), cuya magnitud $\left(\mathrm{M}_{\mathrm{w}} 6,3\right)$ ha sido considerada de los mayores eventos disparados por influencia de una represa (Gupta, 2002). La sismicidad en los alrededores de esta represa ha sido relevante dentro del estudio de la sismicidad disparada, dado que comenzó a crecer significativamente después de su llenado (1961), generándose varios miles de eventos que han incluido aproximadamente 200 sismos ML > 4,0 y 22 sismos ML > 5,0 (Gupta et al., 2015).

La literatura identifica los eventos sísmicos detonados por represas bajo dos condiciones de respuesta principales: rápida y retardada (Simpson et al., 1988). La sismicidad de respuesta rápida suele ocurrir en las proximidades de la represa a una profundidad focal considerada superficial (menor a los $10 \mathrm{~km}$ ) y se caracteriza generalmente por sismos de baja magnitud. La sismicidad de respuesta retardada se genera años después de entrar en operación la represa y se relaciona con los procesos de filtración de agua en profundidad; generando sismos de mayor magnitud comparados con los de respuesta rápida y a profundidades focales que van hasta los $30 \mathrm{~km}$. No obstante, la sismicidad disparada por embalses suele generarse por la combinación de ambos procesos (González de Vallejo et al., 2005).

Un estudio realizado en la represa de las Tres Gargantas (China), encontró que un sismo de baja magnitud ( $M$ $0,9)$ registrado en octubre de 2008, seguido de un evento de magnitud M 4,1 en noviembre de 2008, dos meses después que finalizara su llenado, fueron considerados como sismos de respuesta rápida o inicial. Durante el año 2013, cuatro eventos de magnitud $M>4,0$ ocurrieron durante la etapa de descarga del embalse, sismicidad atribuida a la filtración del agua por un largo período; enmarcándose este tipo de sismicidad como de respuesta retardada o prolongada (Zhang et al., 2018).

Casos de sismicidad disparada por represas también han sido estudiados en Latinoamérica, destacando el estudio de la secuencia sísmica de El Cuchillo (más de 120 sismos $2,8 \leq M_{D} \leq 4,5$, localizados entre 1 y 20 $\mathrm{km}$ de profundidad), ocurrida entre octubre de 2013 y julio de 2014, en la cuenca de Burgos al noreste de México (Montalvo-Arrieta et al., 2018). El estudio señala que el factor detonante de esta sismicidad puede estar relacionado con el cambio del volumen de agua del embalse El Cuchillo, como consecuencia de 3 años de sequía que vaciaron la represa y su rápido proceso de llenado después de la ocurrencia del Huracán Ingrid (2013), lo que propició un incremento de su capacidad cercano al $40 \%$ de su volumen en unos pocos días.

La literatura técnica consultada previo al desarrollo del presente artículo, ha permitido establecer que este tipo de investigación no se ha adelantado en el país o en su defecto, puede encontrarse actualmente en curso y aún no se han publicado sus resultados, razón por la cual, el presente documento espera convertirse en referente y línea base para abordar la temática de sismicidad antropogénica inducida (o disparada) por represas destinadas al almacenamiento de agua a nivel nacional, además de incentivar el desarrollo de futuros análisis sobre esta temática en el país.

\section{Metodología}

El proceso metodológico llevado a cabo dentro de la investigación, así como la información empleada para estos análisis se presenta a continuación.

\section{Selección de represas}

Colombia cuenta actualmente con más de treinta (30) represas distribuidas en diversos puntos de su geografía (Palacios, 2013). Los principales propósitos para su construcción obedecieron a la generación de energía eléctrica, el abastecimiento de agua potable y el riego. Después de evaluar sus características técnicas, se seleccionaron cuatro embalses cómo áreas objeto de estudio (Tabla 1 y Figura 1), destacando como criterio de priorización su capacidad de almacenamiento de agua, condición relacionada en la literatura técnica como uno de los principales factores que se asocian con la generación de sismicidad antropogénica (Consorcio Generación Ituango, 2011; Barros et al., 2018). 
Tabla 1. Represas seleccionadas para la investigación.

\begin{tabular}{cccccc}
\hline Represa & $\begin{array}{c}\text { Ubicación } \\
\text { (Departamento) }\end{array}$ & $\begin{array}{c}\text { Inicio Llenado } \\
\text { (Año) }\end{array}$ & $\begin{array}{c}\text { Volumen Aprox. } \\
\mathbf{x 1 0}^{\mathbf{6}} \mathbf{~ m}^{\mathbf{3}}\end{array}$ & $\begin{array}{c}\text { Superficie Máx } \\
\text { (Ha) }\end{array}$ & $\begin{array}{c}\text { Profundidad } \\
\text { Máx (m) }\end{array}$ \\
\hline Topocoro & Santander & 2014 & 4800 & 6960 & 190 \\
Betania & Huila & 1986 & 1971 & 7400 & 90 \\
Salvajina & Cauca & 1985 & 906 & 2310 & \\
Calima & Valle del Cauca & 1966 & 580 & 2000 & 98 \\
\hline
\end{tabular}

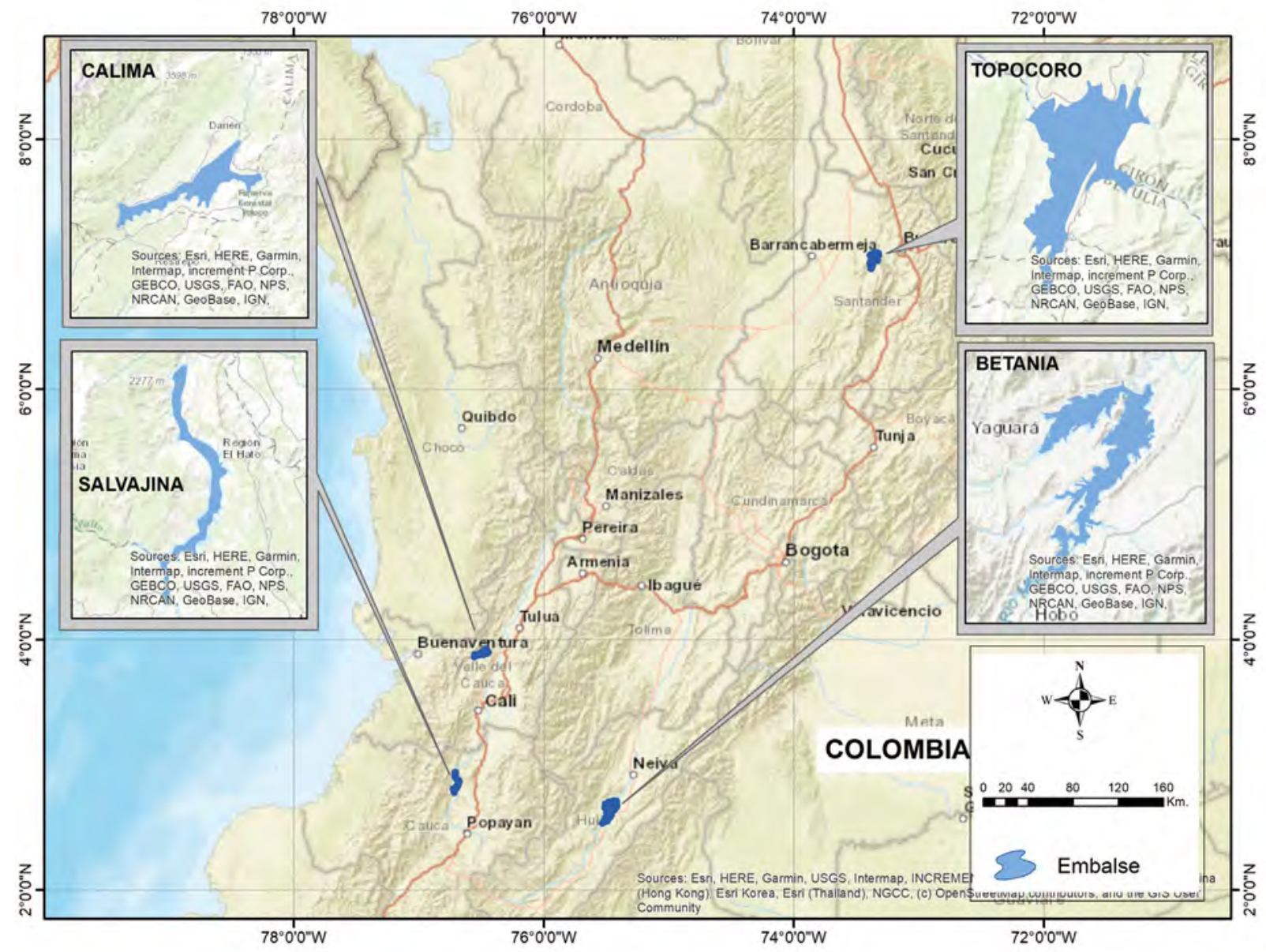

Figura 1. Ubicación general de las represas seleccionadas en la investigación.

\section{Eventos sísmicos}

Los eventos sísmicos registrados en el área circundante a las represas de estudio fueron obtenidos con base en la información disponible consultada de forma pública a través de la página web de la Red Sismológica Nacional de Colombia (RSNC). Para esta búsqueda se definió un límite de $50 \mathrm{~km}$ a partir del centro de la represa (mediante consulta cuadrada) y un máximo de profundidad de $50 \mathrm{~km}$. De esta consulta se obtuvo información sobre fecha y hora de los eventos, coordenadas de ubicación (latitud, longitud y profundidad), magnitud (ML), así como los errores relacionados con el proceso de localización de los sismos, entre otros; dentro de una ventana temporal comprendida entre los años 2000 y 2017. 
En relación con la distribución y cantidad de las estaciones sismológicas pertenecientes a la RSNC, es pertinente señalar que entre los años 2000 y 2015 (como período de referencia) éstas se incrementaron significativamente en el territorio nacional, permitiendo una mejora en el registro de los eventos sísmicos ocurridos en el país; pasando de 17 a 50 estaciones en operación (Figura 2).
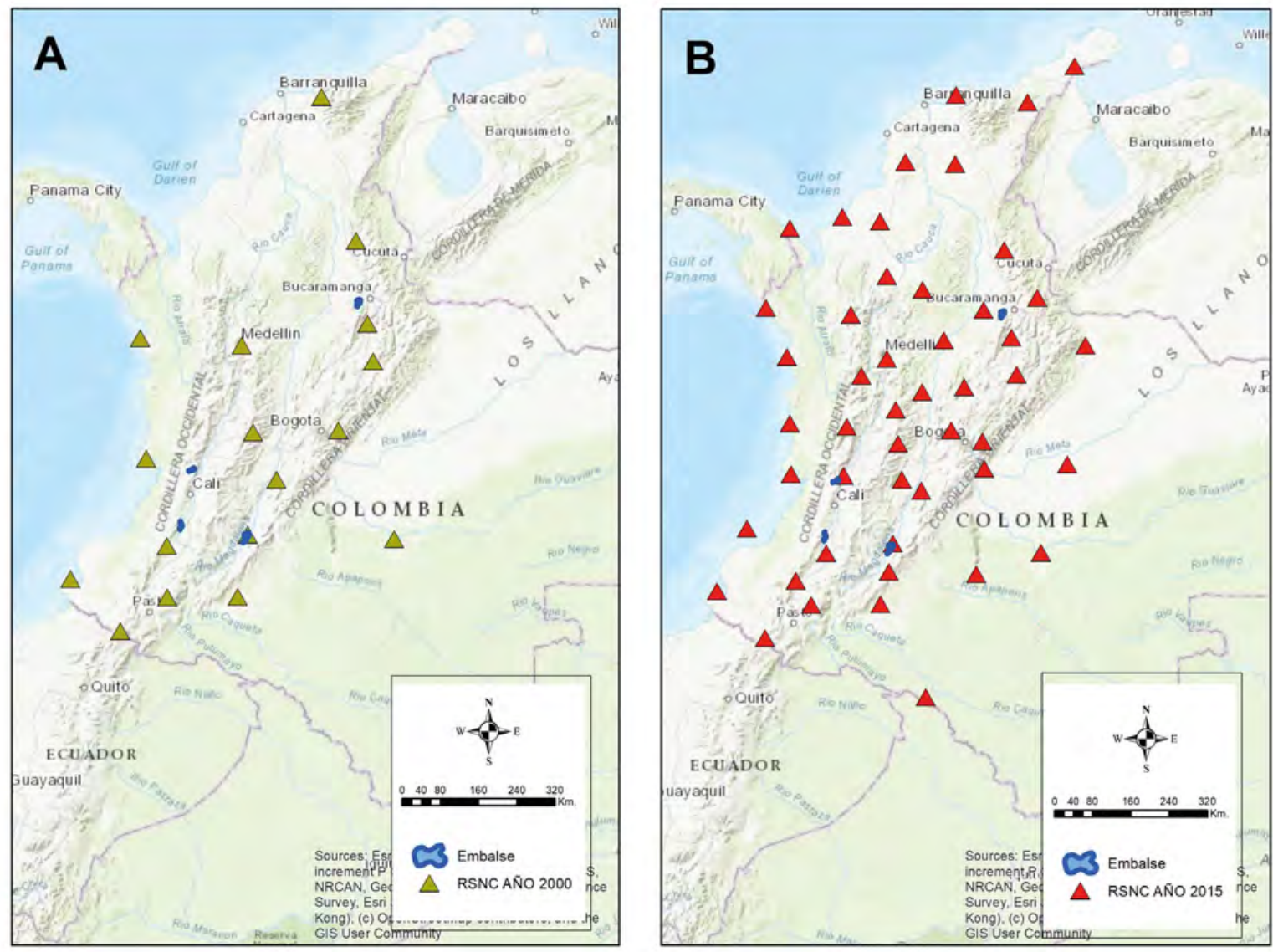

Figura 2. Estaciones de la Red Sismológica Nacional de Colombia (RSNC) en funcionamiento entre: A. Año 2000 y B. Año 2015.

Los umbrales de consulta en área y profundidad definidos en la investigación para la determinación de los eventos sísmicos de interés en los alrededores de cada represa son superiores en relación con otros estudios realizados a nivel mundial. Por ejemplo, para el embalse Song Trank 2 (Vietnam) se delimitó el análisis a un radio de $30 \mathrm{~km}$ (Lizurek et al., 2017); mientras que otros investigadores han optado por realizar sus análisis dentro de límites inferiores a los 20 km (Drakatos et al., 1998; Mikhailov et al., 2017; Stiros y Pytharouli, 2018).

\section{Volúmenes de agua de las represas}

Para el análisis de los volúmenes del agua acumulada en cada una de las represas, estos fueron obtenidos a través de la página web de la firma XM, entidad filial de la empresa ISA, reconocida compañía a nivel nacional relacionada con la transmisión de energía eléctrica.

\section{Relación Gutenberg-Richter (G-R)}

Para cada área de estudio se determinó la relación G-R, cuyo modelo estadístico establece una correlación entre 
la magnitud de los eventos sísmicos y su distribución acumulada de ocurrencia. Este modelo, establecido por Gutenberg y Richter en 1944, puede ser expresado mediante formulación exponencial (Urban et al., 2016) presentada en la ecuación 1.

$$
\mathrm{N}=10^{(\mathrm{a}-\mathrm{b} * \mathrm{M})}
$$

Donde $\mathrm{N}$ representa el número de eventos de magnitud mayor o igual a M, y los parámetros “a” y "b”, son constantes relacionadas con el ejercicio matemático de regresión.

\section{Criterio de filtrado de los eventos sísmicos}

Para seleccionar los eventos sísmicos con la mejor localización se decidió utilizar como criterio de filtrado los valores correspondientes a los errores de latitud, longitud y profundidad, definiendo para ello un umbral máximo de $10 \mathrm{~km}$; proceso previo al análisis de los eventos en relación con su distribución en magnitud, profundidad y ocurrencia anual.

\section{Análisis de esfuerzos bajo la teoría de Boussinesq}

La posible influencia de la sobrecarga hidrostática ejercida por los embalses frente a la ocurrencia de los eventos sísmicos fue analizada con base en la relación matemática propuesta por Joseph Boussinesq en 1885 (Das, 2012) dentro del desarrollo de su teoría de esfuerzos y deformaciones; teoría empleada en investigaciones similares a nivel internacional como en Koyna-Warna, India (Gahalaut et al., 2018) y en el Himalaya (Bettinelli et al., 2008).

La expresión empleada permite encontrar el incremento de esfuerzo vertical $(\Delta \sigma \mathrm{z})$ para cualquier punto debajo del centro de una carga circular de radio $\mathrm{R}$, sometida a una presión de contacto (q) uniformemente distribuida, y para una profundidad (Z) de interés cualquiera (Ecuación 2).

$$
\Delta \sigma \mathrm{z}=\mathrm{q} *\left[1-\left[\frac{1}{1+\left(\frac{\mathrm{R}}{\mathrm{z}}\right)^{2}}\right]^{3 / 2}\right]
$$

El límite inferior de referencia corresponderá al 10\% del esfuerzo aplicado $(\Delta \sigma z=0,10 q)$ por la sobrecarga, valor por debajo del cual las presiones transmitidas en profundidad son tan pequeñas como para ser consideradas de interés (González de Vallejo et al., 2006).

\section{Eventos sísmicos analizados bajo la teoría de Boussinesq}

Este análisis se centró en sismos con magnitud $\mathrm{M}_{\mathrm{L}} \geq 3,0$ cuyo umbral es superior a la magnitud de completitud de la relación G-R obtenida del catálogo de eventos de la consulta inicial y cuya energía liberada podría ofrecer alguna condición de riesgo para las poblaciones cercanas. En cualquier caso, esta magnitud es inferior en comparación con otros estudios, donde se han empleado límites $M_{L} \geq 3,5$ (Dahm et al., 2010; Mikhailov et al., 2017; Zhang et al., 2018).

\section{Influencia de eventos sísmicos bajo la teoría de Boussinesq}

Para analizar la influencia de la sobrecarga ejercida por el embalse, se contrastará de forma gráfica la localización de los sismos $M_{L} \geq 3,0$ con el área del bulbo de esfuerzos correspondiente al $10 \%$ de la presión de contacto ejercida en superficie. Los sismos que llegasen a cumplir con este criterio, tendrían una alta posibilidad de ser disparados por la sobrecarga del embalse.

Los sismos analizados serán contrastados con los principales sistemas de fallas existentes en cada zona de estudio, con base en la información publicada por el Servicio Geológico Colombiano (SGC) (Gómez et al., 2015).

\section{Resultados}

Este apartado presenta los resultados más relevantes obtenidos con base en los análisis realizados para las represas de Betania, Topocoro, Salvajina y Calima, priorizando aspectos relacionados con la distribución espacial de los eventos sísmicos en sus alrededores, la relación G-R, y la influencia de la sobrecarga hidrostática del embalse y los sismos registrados empleando la teoría de esfuerzos de Boussinesq y el método de correlación de Pearson.

En relación con la represa de Betania, la mayor cantidad de eventos registrados en sus alrededores ocurrieron al sur del embalse (Figura 3), con una gran concentración de sismos en los primeros $5 \mathrm{~km}$ de profundidad (48,9\% del total de los 1848 eventos filtrados). Se observa que los sismos guardan relación con la orientación de los principales sistemas de fallas identificados en la región (fallas de El Agrado-Betania, de Algeciras y de Rivera). 


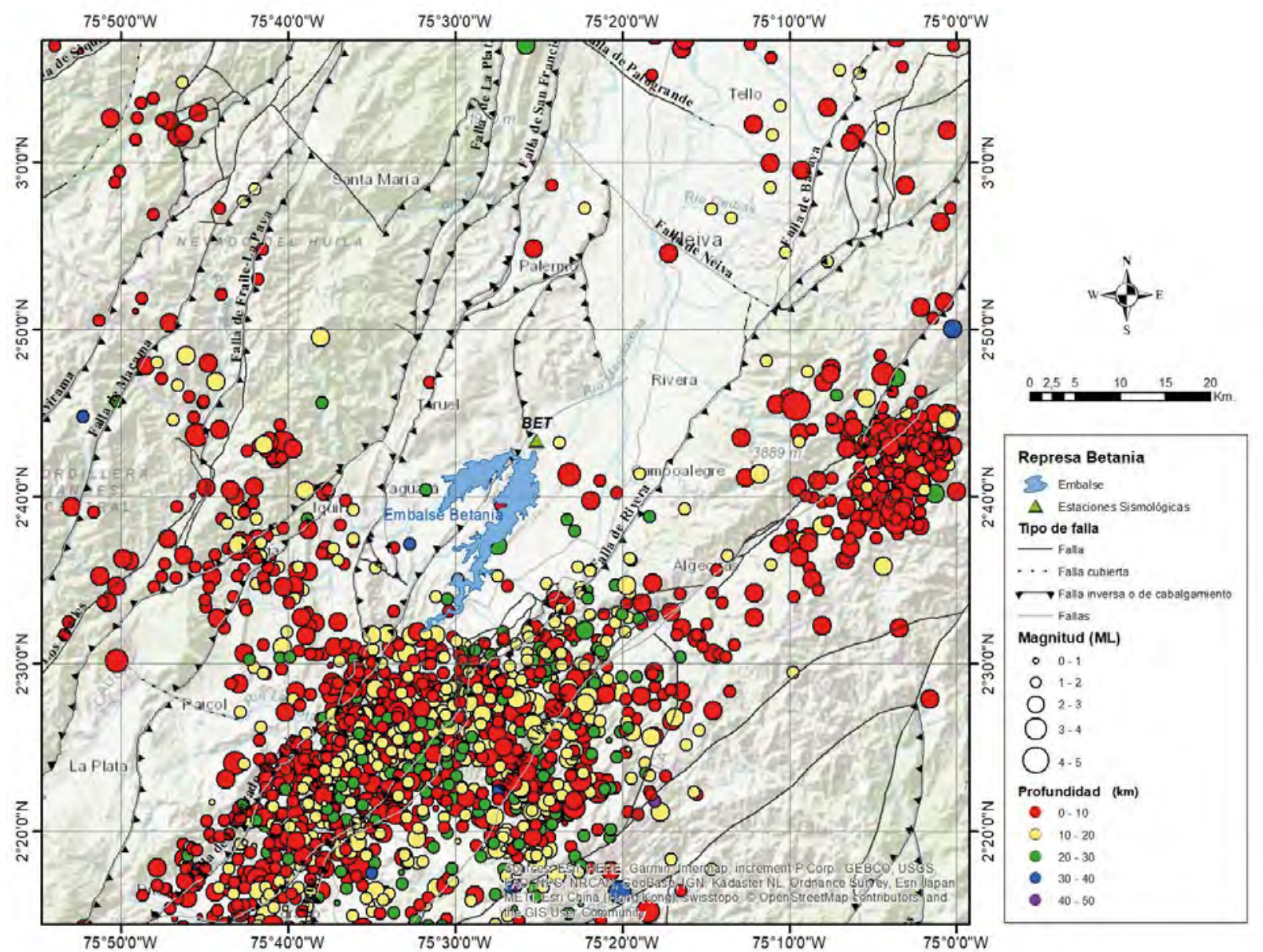

Figura 3. Localización de sismos alrededor de la represa de Betania.

Para la represa de Topocoro los eventos se encuentran más dispersos y retirados del centro del embalse (Figura 4), aunque se mantiene la tendencia de registro de una mayor cantidad de sismos en los primeros $5 \mathrm{~km}$ de profundidad (32,7\% del total de 482 eventos filtrados). En relación con la orientación de los sismos con los sistemas de fallas existentes en cercanía al embalse, algunos se concentran en dirección NE-SW, coincidiendo con las fallas de Arrugas y La Salina, y otros en dirección NW-SE, coincidiendo con la orientación de la falla Bucaramanga-Santa Marta.

La represa de Salvajina registró en sus alrededores un total de 168 sismos que cumplieron el criterio de filtrado, registrando el $53 \%$ de ellos en los primeros $5 \mathrm{~km}$ de profundidad. Debido a los diversos sistemas de fallas existentes en la región, no existe una concentración de eventos que esté gobernada por un sistema en particular (Figura 5).

En relación con la represa de Calima, 617 eventos se registraron después del filtrado (Figura 6), de los cuáles el 36\% se concentró en los primeros $5 \mathrm{~km}$ de profundidad. Frente a su distribución espacial, se observa una ligera concentración de sismos hacia el occidente del embalse que guardan relación con los sistemas de fallas DaguaCalima y de Trujillo. A profundidades mayores a $50 \mathrm{~km}$ (fuera del límite de consulta del estudio), los eventos sísmicos estarían influenciados por deformación de la corteza en la denominada zona de Wadatti-Benioff intermedia (Pérez et al., 2014).

Frente a la relación Gutenberg-Richter (G-R), los resultados del parámetro “b” arrojaron resultados muy cercanos para las inmediaciones de las represas Betania y Calima, existiendo una leve tendencia a la ocurrencia de eventos de mayor magnitud frente a eventos de menor liberación de energía en el entorno de Calima (Figura 7). Para la represa de Salvajina, el parámetro "b” obtenido fue mayor respecto a las dos represas anteriores, ofreciendo una tendencia a la ocurrencia de eventos sísmicos de menor magnitud. 


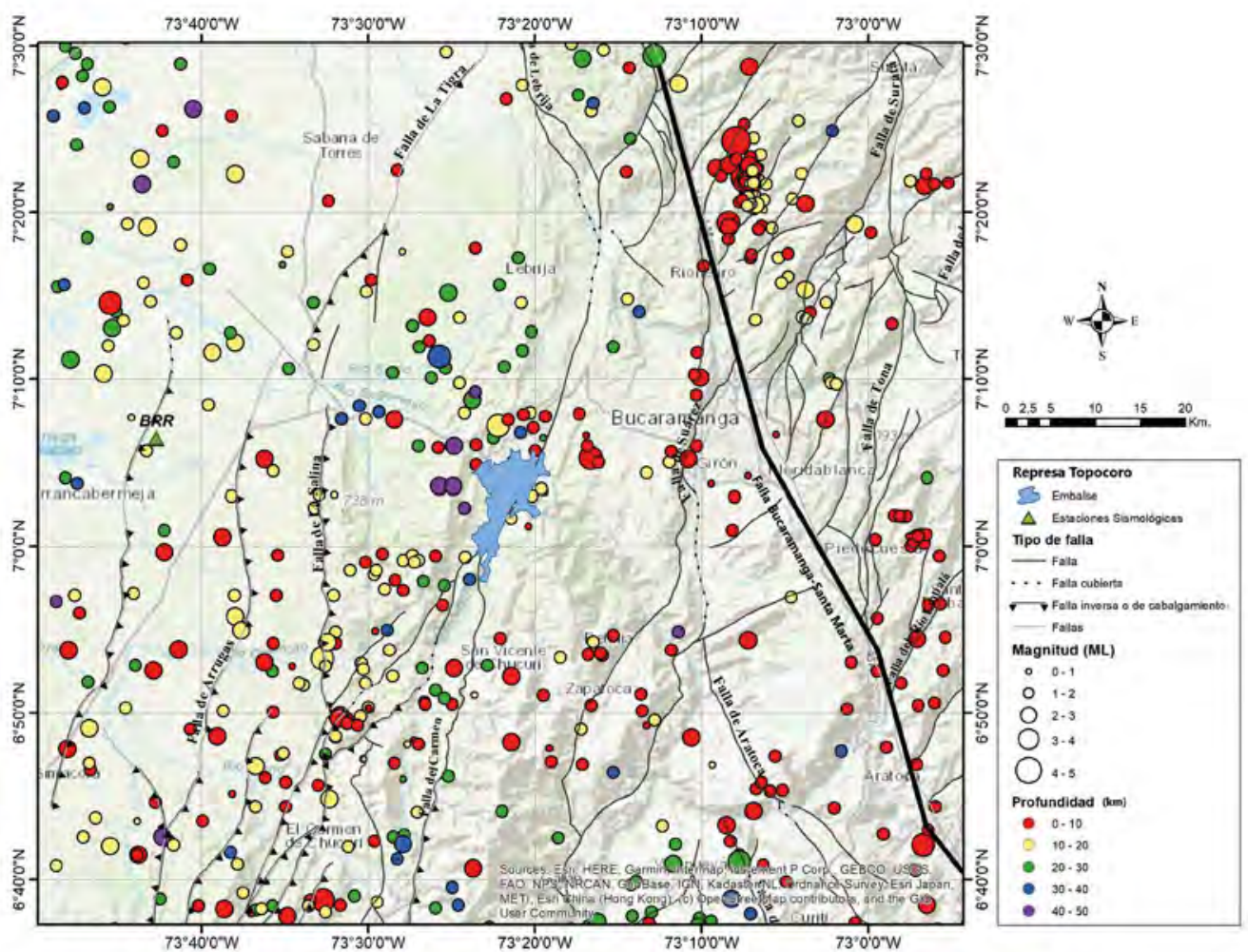

Figura 4. Localización de sismos alrededor de la represa de Topocoro.

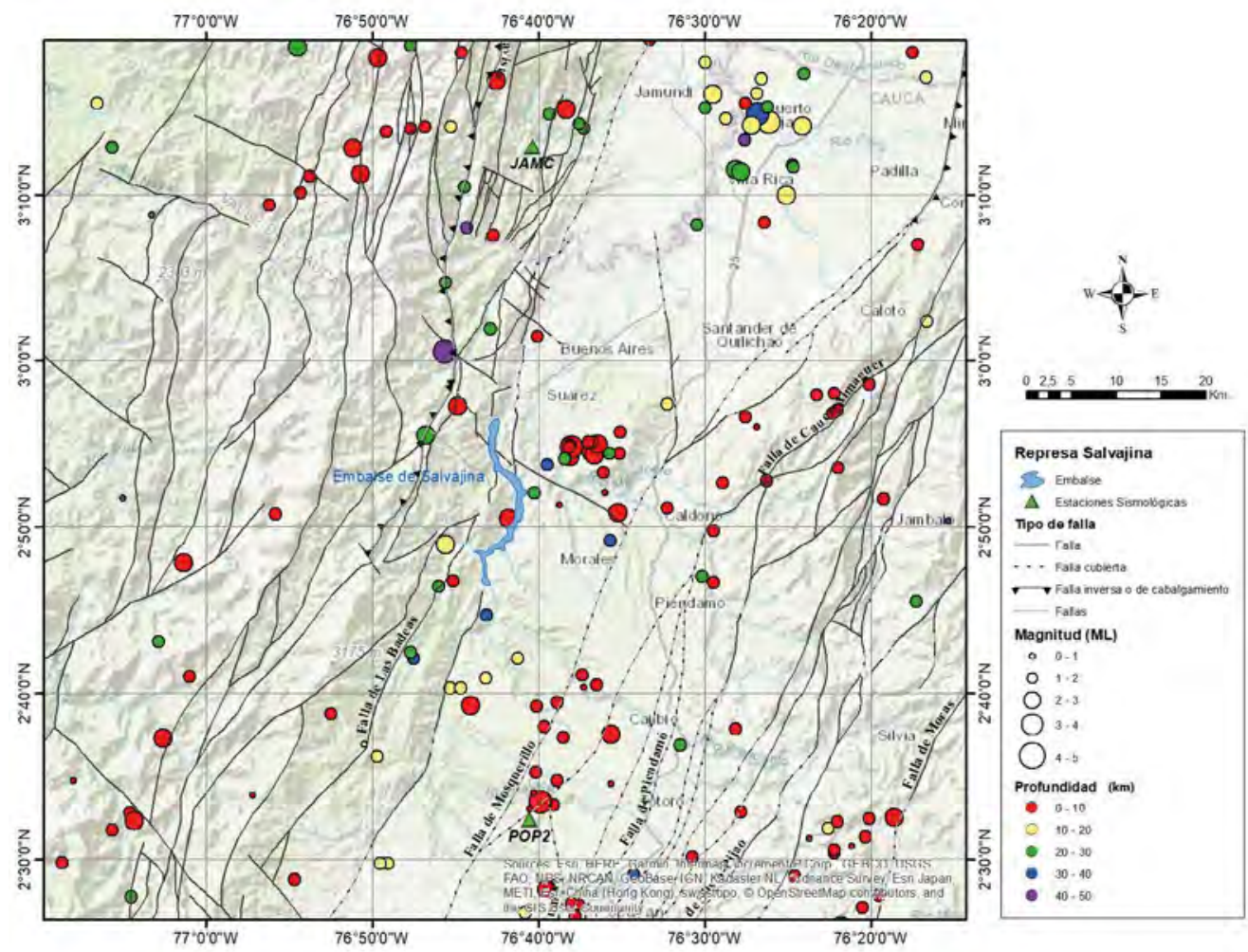

Figura 5. Localización de sismos alrededor de la represas de Salvajina. 


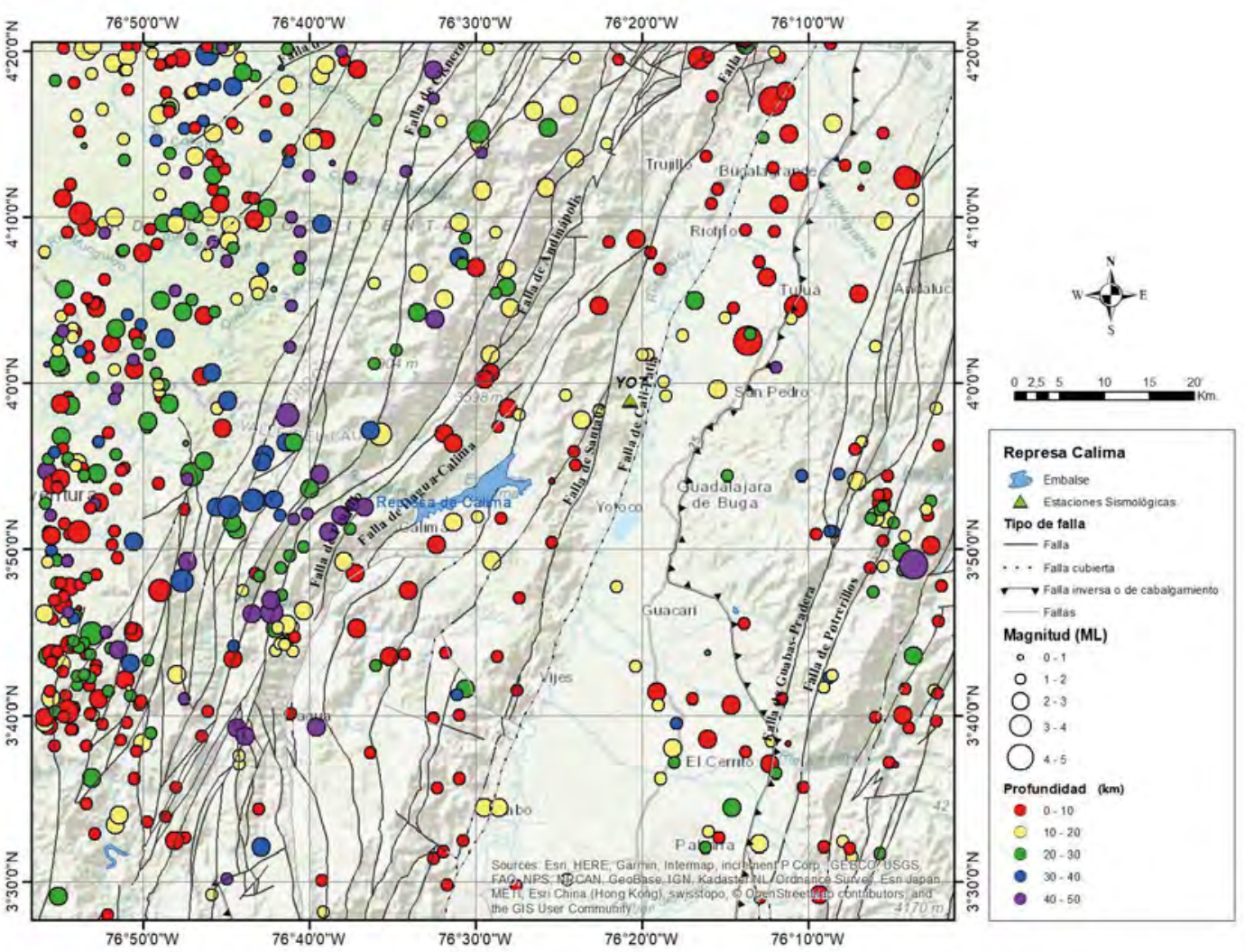

Figura 6. Localización de sismos alrededor de la represa de Calima.
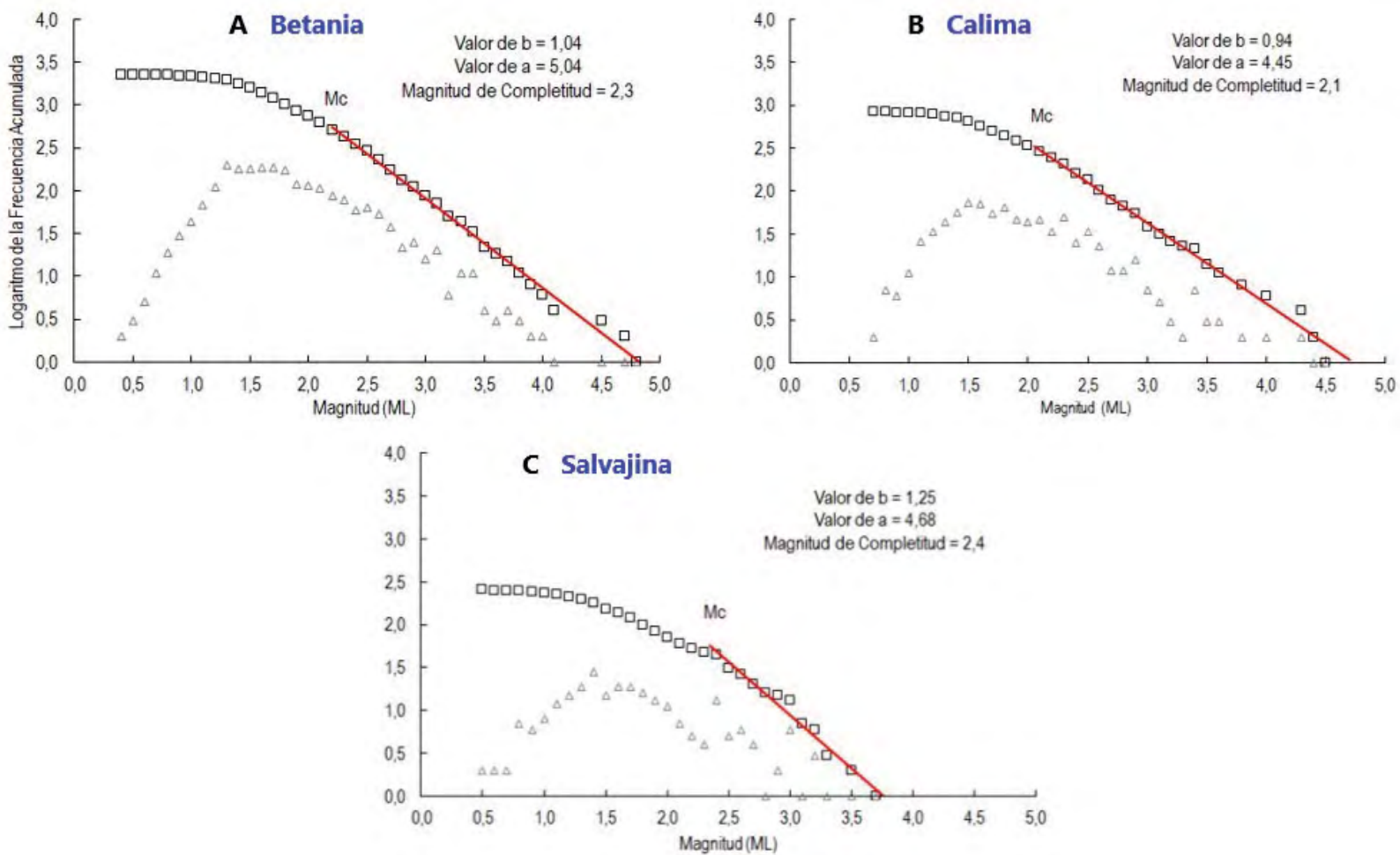

Figura 7. Relación G-R para el área cercana a las represas de: A. Betania, B. Calima y C. Salvajina. 
Para la represa de Topocoro, es posible comparar el parámetro "b" antes y después de su entrada en operación. Aunque en ambos casos el valor de "b" es menor a 1,0, existe un leve incremento de este parámetro después de construir la represa e iniciar el proceso de sobrecarga hidrostática, es decir, una leve tendencia a la ocurrencia de eventos de menor magnitud (Figura 8).
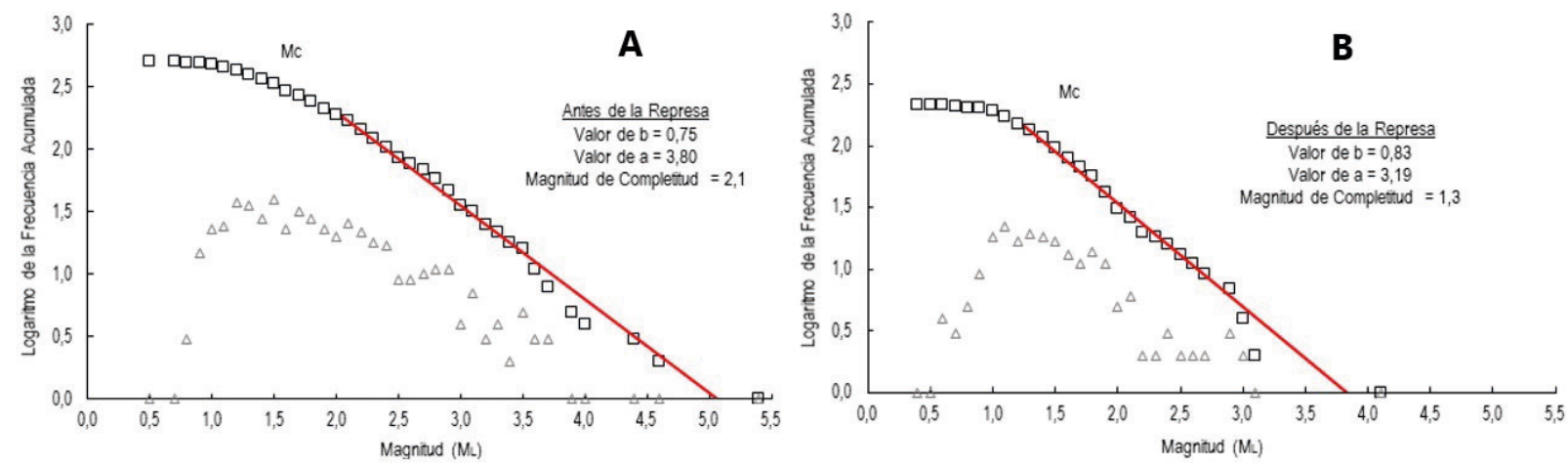

Figura 8. Relación G-R para el entorno cercano a la represa de Topocoro: A. Antes de la represa y B. Después de la represa.

Como parte del análisis de Boussinesq realizado para la represa de Betania, se encontró un evento que podría estar influenciado por la sobrecarga del embalse, no obstante, su ubicación ligeramente por fuera de los límites del bulbo de esfuerzos, no es suficiente para establecer su relación directa (Figura 9). Para la represa de Topocoro, aunque se localizan dos sismos muy cerca de la zona de influencia, no coinciden simultáneamente dentro del bulbo de esfuerzos (Figura 9).

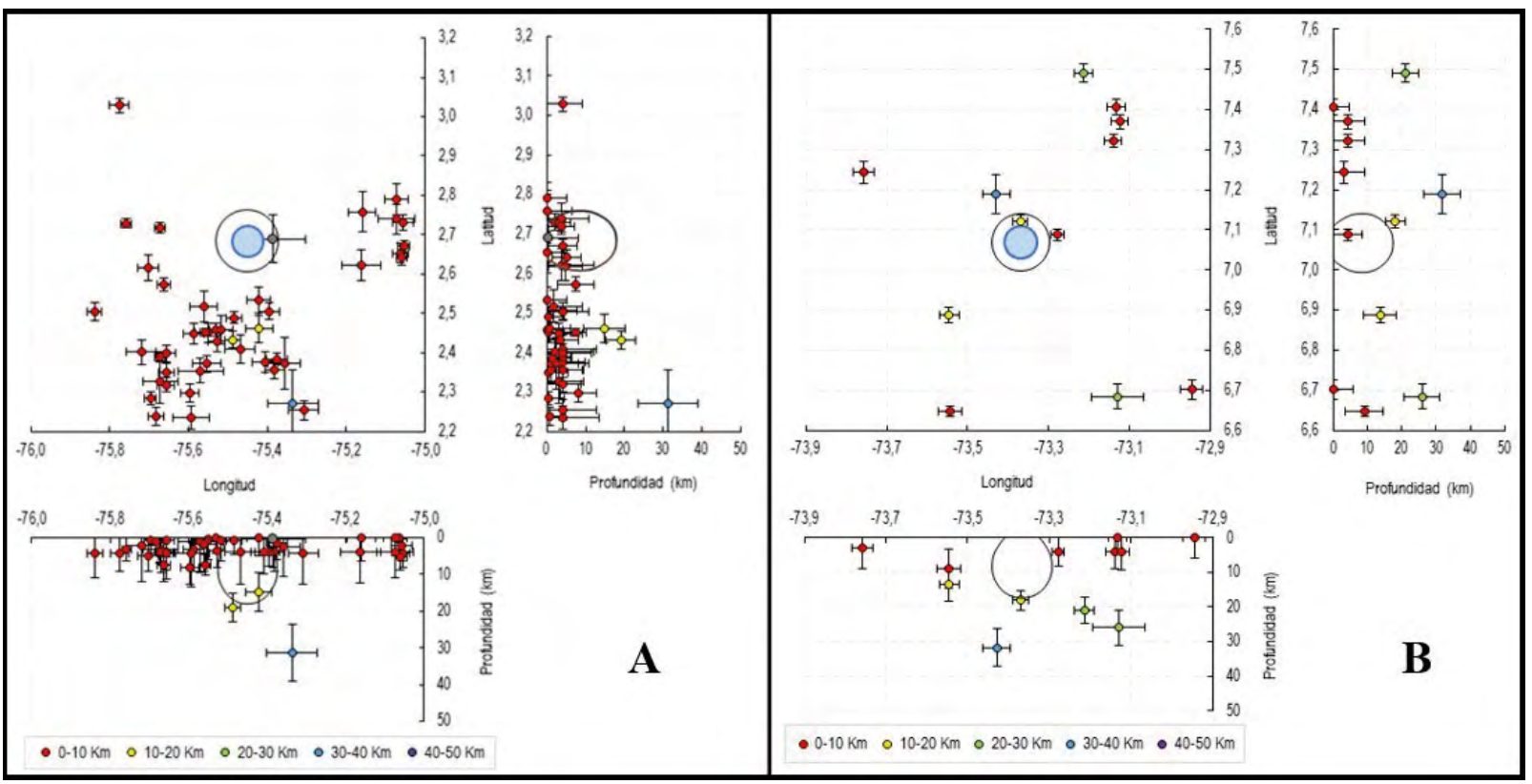

Figura 9. Representación de eventos sísmicos bajo la influencia de la sobrecarga hidrostática para los embalses de: A. Betania y B. Topocoro. 
Los análisis para las represas de Calima y Salvajina no arrojaron resultados correlacionables con la influencia de la sobrecarga ejercida por cada una de ellas (Figura 10), destacando que, para ellas, el proceso de filtrado de sismos con magnitud $\mathrm{M}_{\mathrm{L}}>3,0$, redujo considerablemente el número de eventos sísmicos con los cuáles se realizó el análisis.

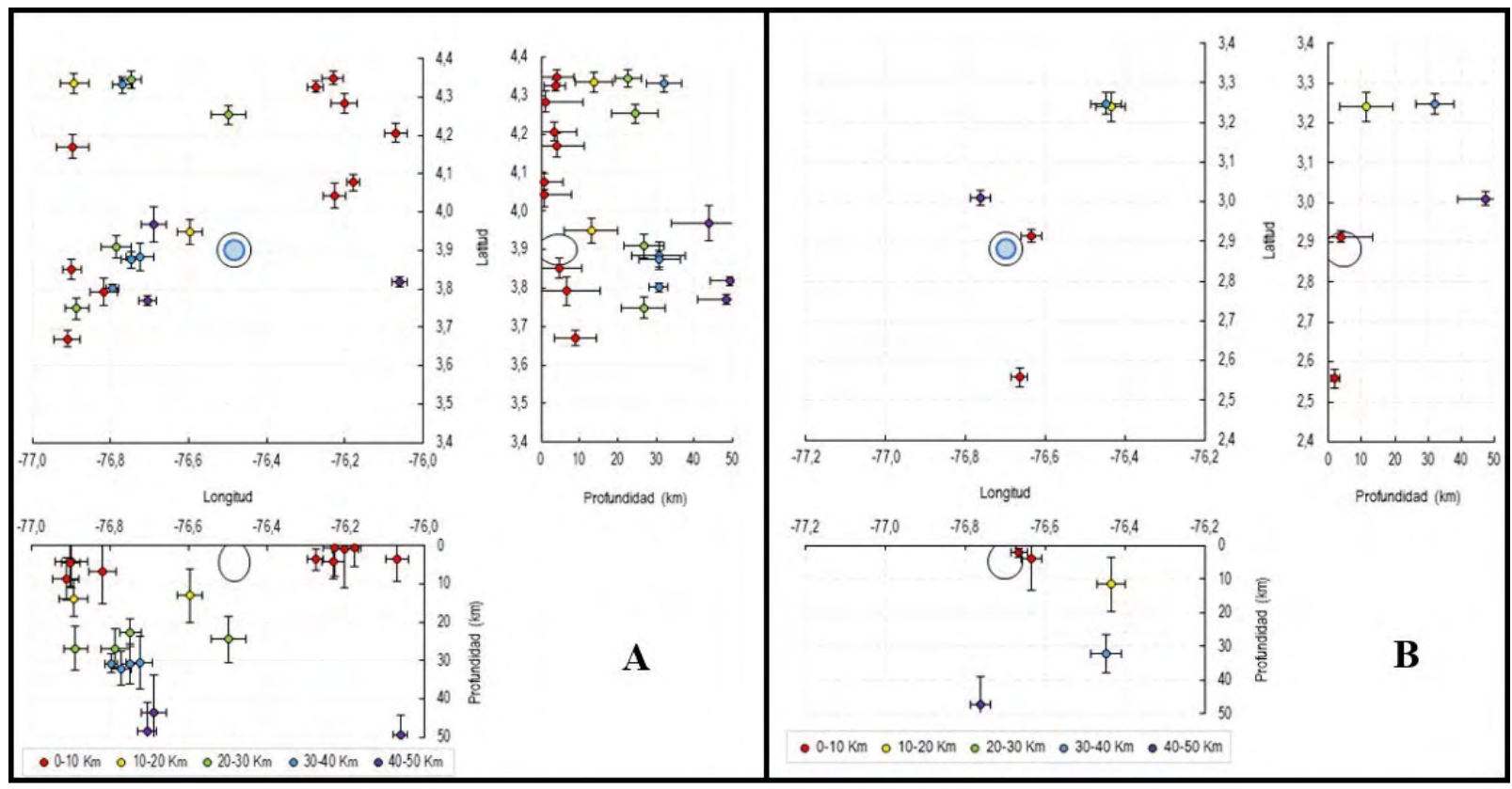

Figura 10. Representación de eventos sísmicos bajo la influencia de la sobrecarga hidrostática para los embalses de: A. Calima y B. Salvajina.

Para analizar la actividad sísmica registrada en los alrededores de cada represa contra el volumen de agua almacenada, se empleó el método de correlación de Pearson como prueba primaria para establecer una relación entre estas variables (Zhang et al., 2017). Producto de este análisis, se observó que existe una gran variación en los resultados de los coeficientes de correlación obtenidos en torno a cada represa, los cuales oscilaron de mayor a menor correlación de la siguiente forma: moderada inversa $(\mathrm{R}=-0,57)$ para la represa de Betania (Figura 11), baja directa $(\mathrm{R}=0,36)$ para la represa de Topocoro (Figura 12), muy baja directa $(\mathrm{R}=0,14)$ para la represa de Salvajina (Figura $13)$, y nula $(\mathrm{R}=-0,07)$ para la represa de Calima.

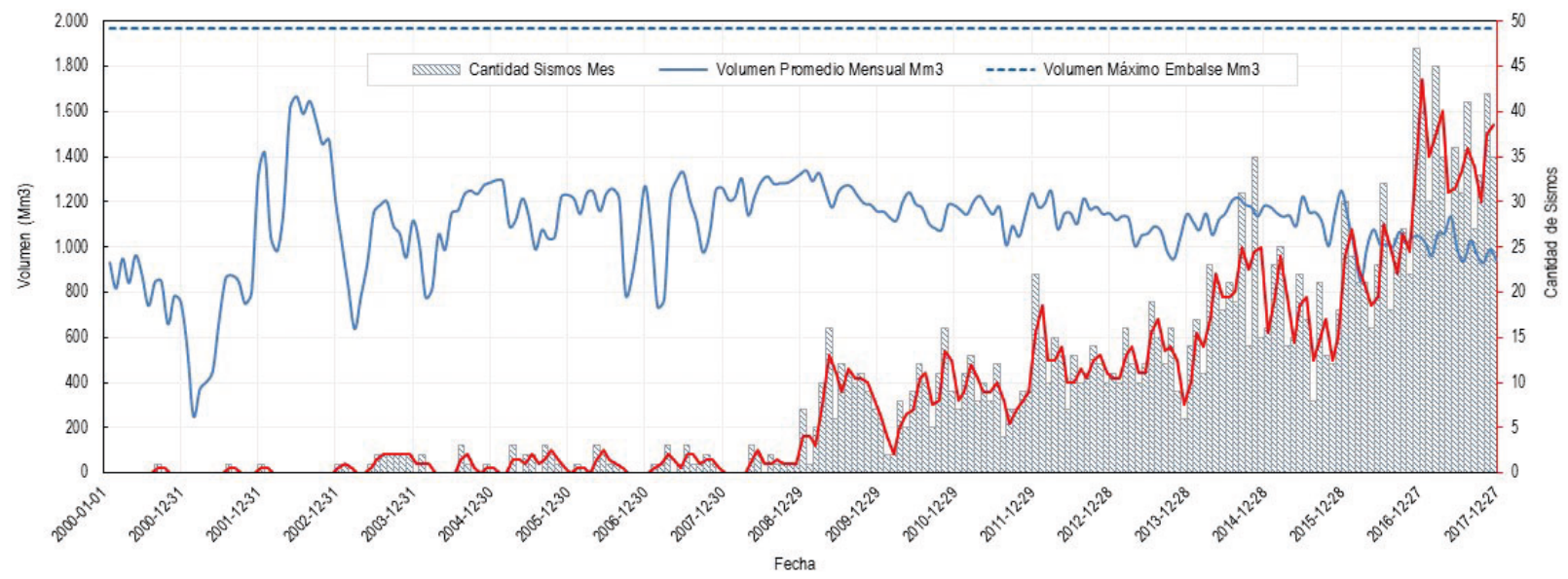

Figura 11. Comparativo mensual entre la variación del volumen del embalse de Betania y la sismicidad registrada en sus alrededores, período 2000 - 2017. 


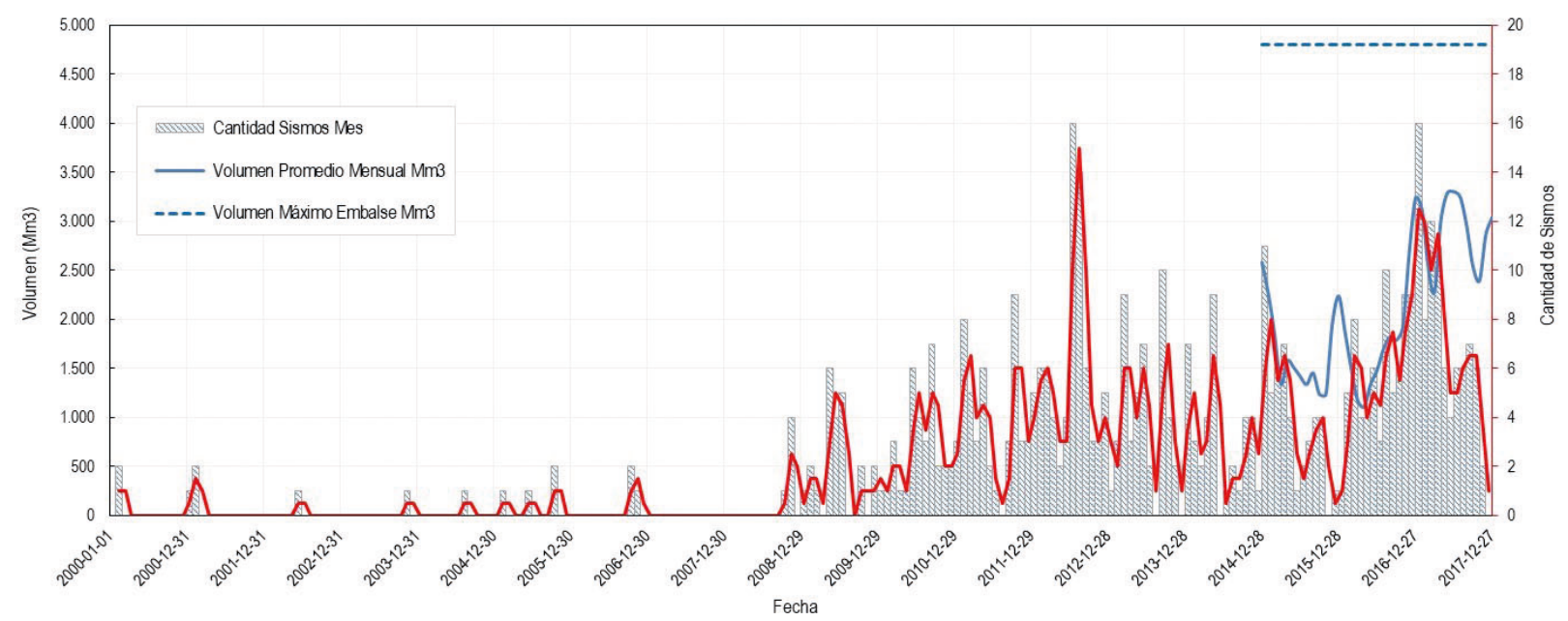

Figura 12. Comparativo mensual entre la variación del volumen del embalse de Topocoro y la sismicidad registrada en sus alrededores, período 2000 - 2017.

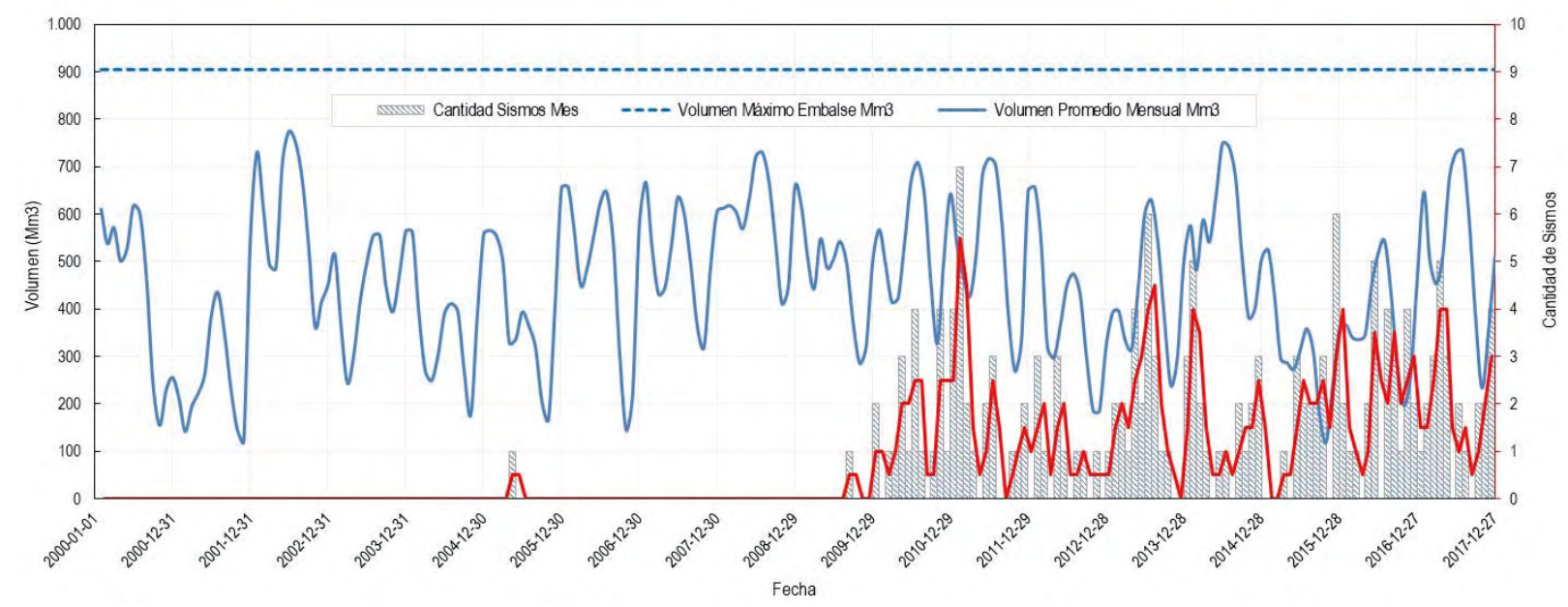

Figura 13. Comparativo mensual entre la variación del volumen del embalse de Salvajina y la sismicidad registrada en sus alrededores, período 2000 - 2017.

\section{Discusión}

El criterio de filtrado utilizado para seleccionar los eventos sísmicos con la mejor localización determinó que, el parámetro "error de profundidad” direccionó esta selección por encima de los errores de latitud y longitud; reflejando una menor reducción de registros para las represas de Betania y Calima, en comparación con la represa de Urrá (por su baja cantidad de datos no fue incluida en el presente artículo), tal como se observa en la Figura 14.

En relación con las magnitudes y profundidades de los sismos registrados alrededor de las represas (después de filtrados), aproximadamente el 95\% de los eventos son de baja liberación de energía $\left(M_{L} \leq 3,0\right)$ y más del $75 \%$ pueden ser categorizados como sismos superficiales $(<25 \mathrm{~km}$; Figura 15).

Aunque en investigaciones nacionales (FlórezHurtado et al., 2013; Muñoz-Burbano et al., 2015) e internacionales (Haggag et al., 2009; Lizurek et al., 2017; Zhang et al., 2018), se han definido modelos de velocidad con el fin de mejorar la localización (y/o relocalizar) de los eventos sísmicos, el presente trabajo no incorporó este procesamiento, y empleó la información de referencia obtenida mediante consulta a la página web de la RSNC, reconociendo la calidad y limitaciones de los datos. 
El parámetro "b" de la relación G-R refleja para las represas analizadas, con excepción de Topocoro y Calima, una leve tendencia a la ocurrencia de eventos de baja magnitud (b>1,0); sin embargo, los análisis para Topocoro después de la construcción de la represa ofrecen un leve incremento en este parámetro, lo que podría relacionarse con una menor capacidad del entorno para almacenar esfuerzos y generar sismos con liberaciones de energía de baja magnitud (Figura 16).

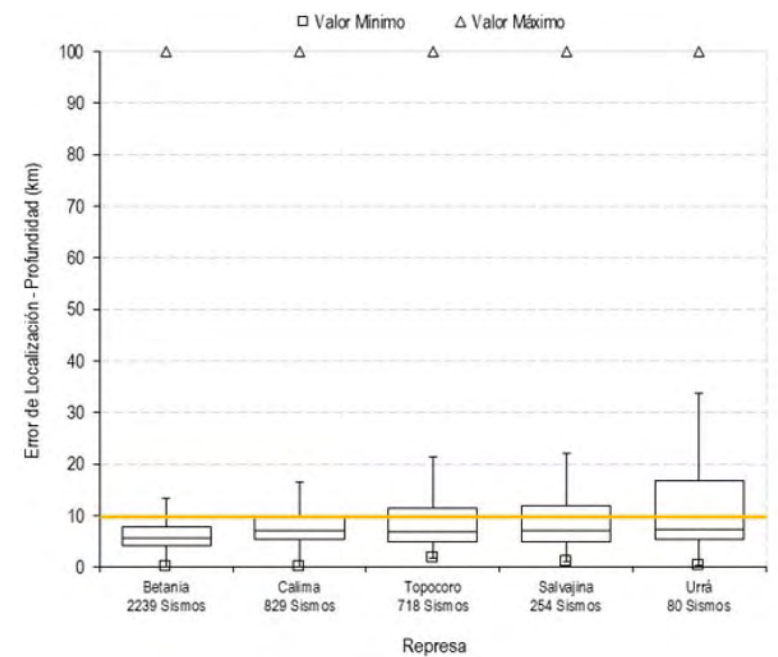

$\mathbf{A}$

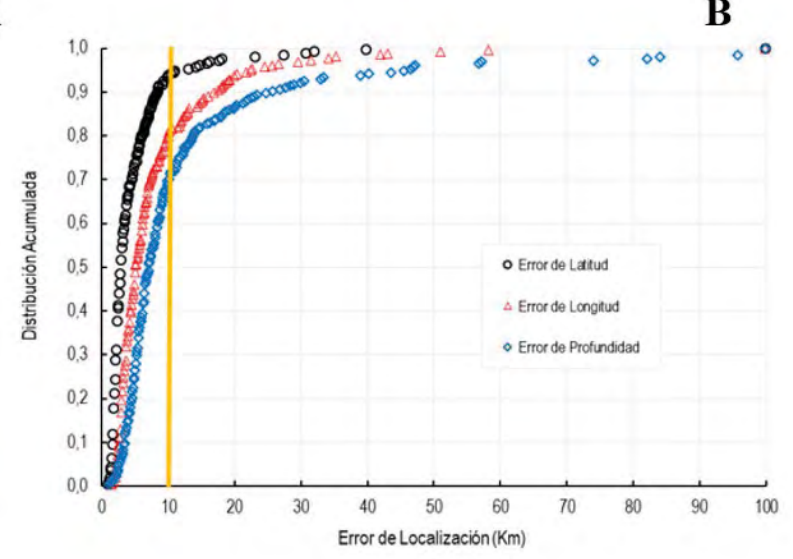

Figura 14. A. Distribución de error de localización por profundidad mediante diagrama de Boxplot para diversas represas y B. Distribución de errores de localización para la represa de Salvajina.
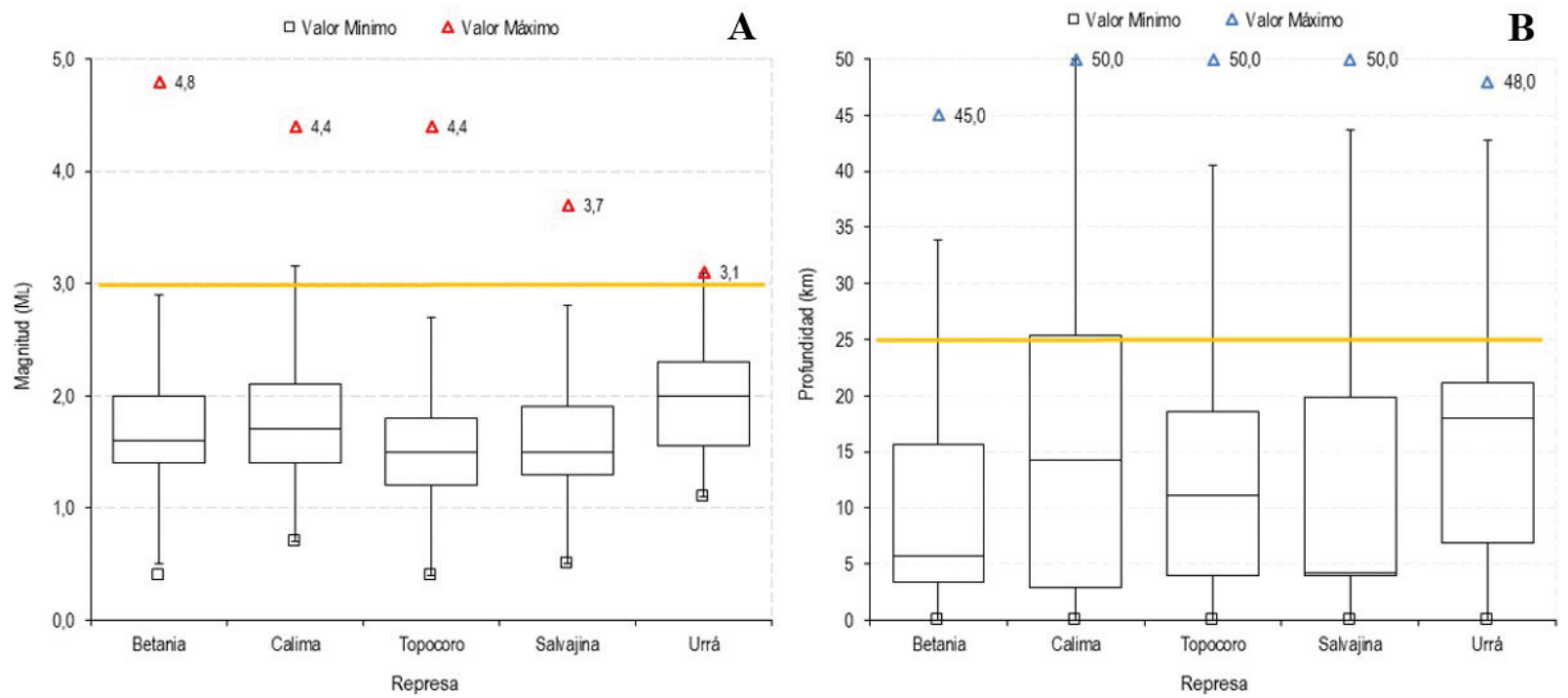

Figura 15. Distribución de eventos sísmicos registrados alrededor de las represas analizadas mediante diagrama de Boxplot: A. Magnitud y B. Profundidad.

En relación con los eventos sísmicos ocurridos en los alrededores de las represas entre los años 2000 y 2017, es importante analizar que para cada una de ellas se observó un incremento en la cantidad de sismos registrados a partir de los años 2008 y 2009, el cual, aunque no ofrece la misma tasa de crecimiento para todas las represas si guarda una coherencia con el incremento en la cantidad de instrumentos sísmicos que fueron puestos en operación por la RSNC a partir del año 2008, lo que mejoró la detección y localización de los sismos en estas regiones, tal como se observa en la Figura 17. 


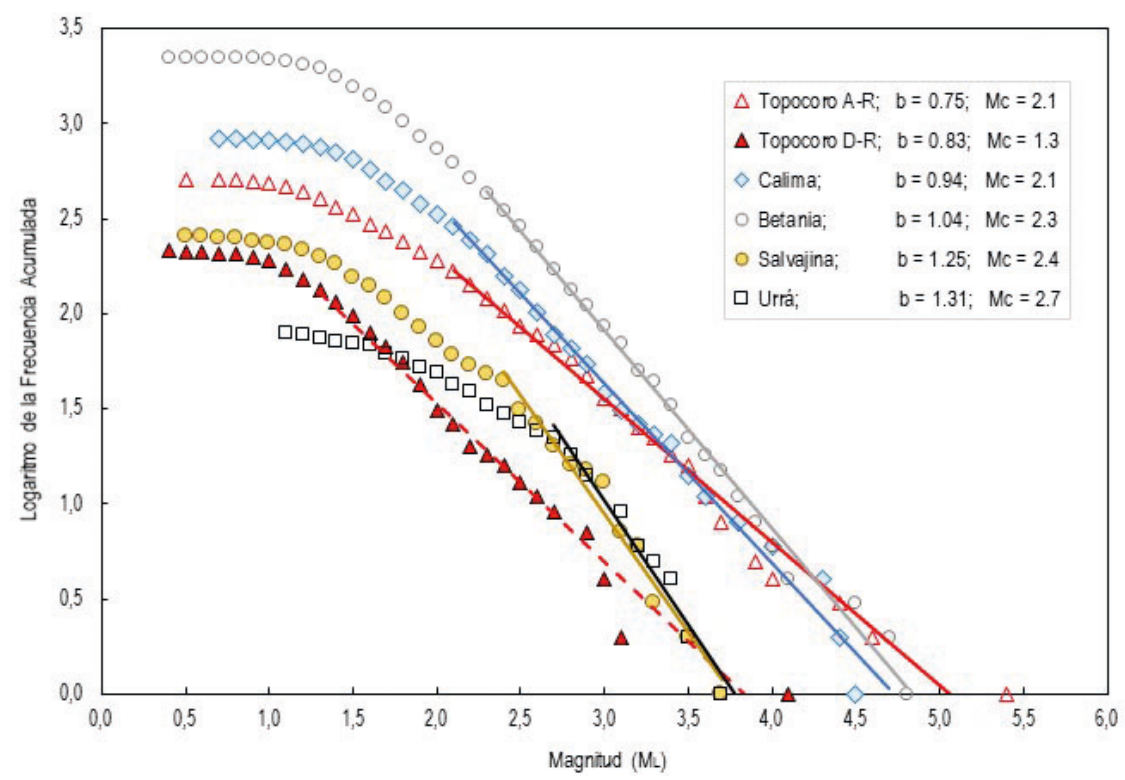

Figura 16. Comparación del parámetro “b” de la relación G-R en los alrededores de las represas estudiadas.

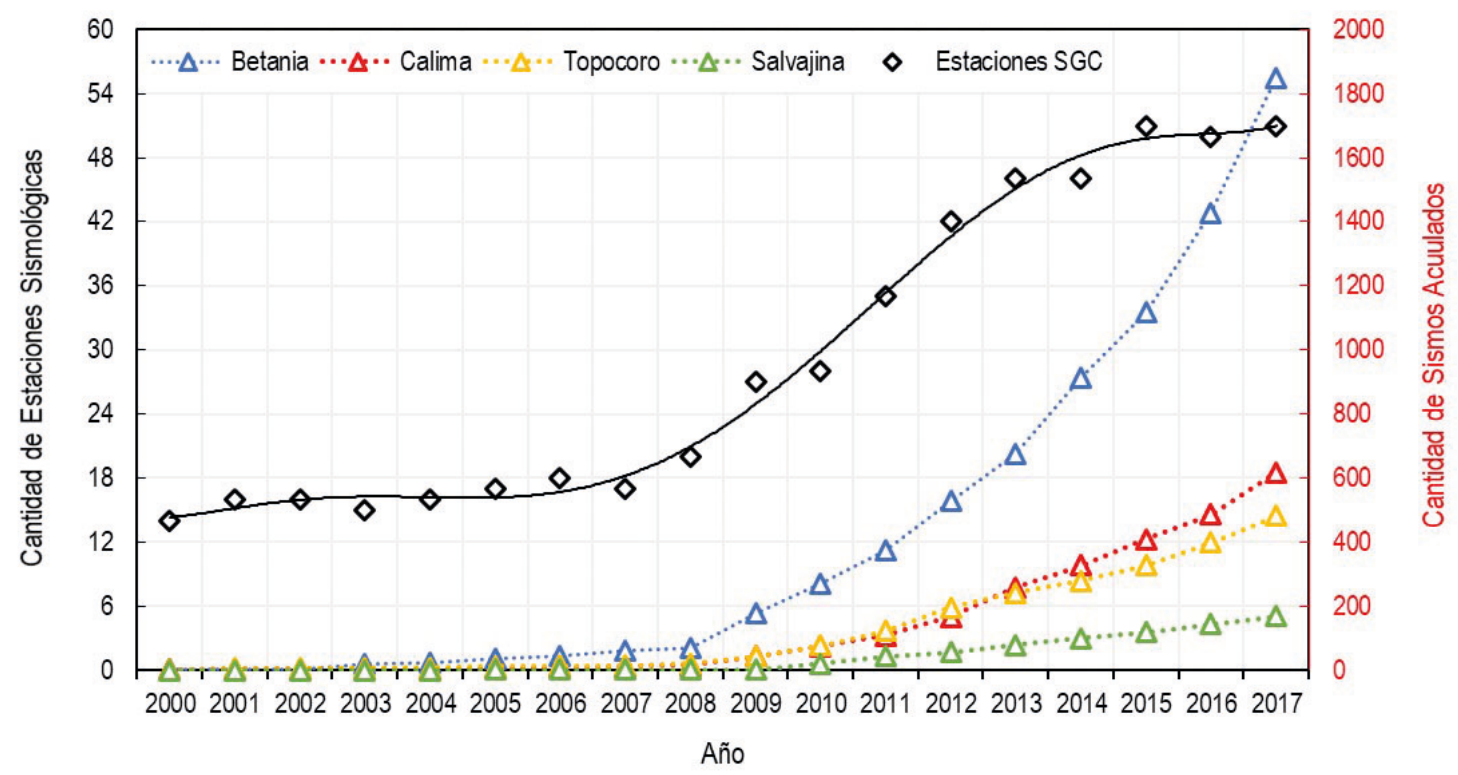

Figura 17. Registro acumulado de eventos sísmicos reportados en los alrededores de las represas de Betania, Calima, Topocoro y Salvajina, así como de la cantidad de estaciones sismológicas de la RSNC en operación durante los años 2000 y 2017.

En los alrededores de las represas de Betania, Calima y Topocoro se registró mayor cantidad de eventos sísmicos en comparación con las represas de Salvajina y Urrá; situación que puede relacionarse con la complejidad tectónica existente en estas regiones (Figura 18). En complemento, este análisis refleja que mayores volúmenes de almacenamiento no inciden necesariamente en la ocurrencia de eventos sísmicos de mayor magnitud.
Al analizar los sismos $\mathrm{ML} \geq 3,0$ registrados cerca a cada represa, pocos eventos se localizan dentro de los primeros $20 \mathrm{~km}$ de distancia y en profundidades menores a los $15 \mathrm{~km}$; límites que suelen relacionarse con eventos antropogénicos disparados (o inducidos) por embalses (Figura 19). No obstante, análisis realizados para la represa de Topocoro, teniendo en cuenta los eventos registrados en los primeros $5 \mathrm{~km}$ de profundidad (después del proceso de filtrado), 
evidencian un aumento en la cantidad de eventos ocurridos después de entrar en operación el embalse, $\sin$ que existiera una variación significativa en la cantidad de estaciones sismológicas en operación durante el período 2015 a 2017 (Figura 17).

De forma complementaria, al comparar los eventos sísmicos registrados de forma acumulada antes (2000-
2014) y después (2015-2017) de la represa de Topocoro, se encontró con posterioridad a su construcción e inicio de operación, una mayor pendiente en la línea de tendencia para estos eventos, situación que podría reflejar cierta influencia ejercida por la sobrecarga hidrostática impuesta por el embalse (Figura 20).

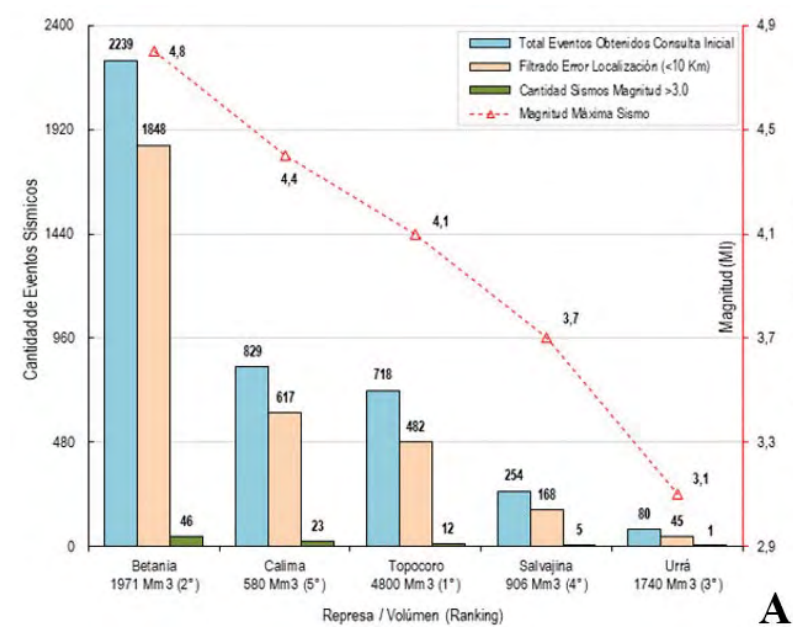

Figura 18. A. Cantidad de sismos registrados en los alrededores de cada represa y de ellas.

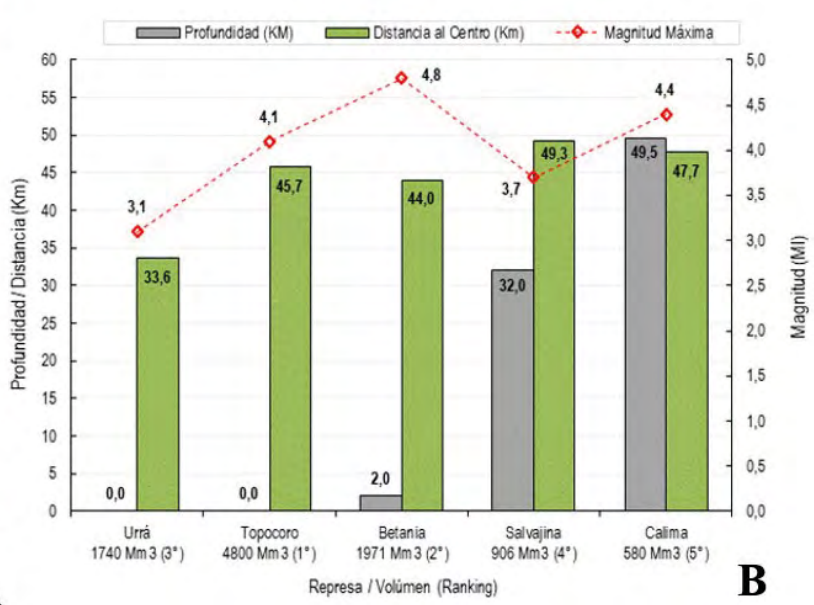

B. Mayores registros sísmicos en cada uno
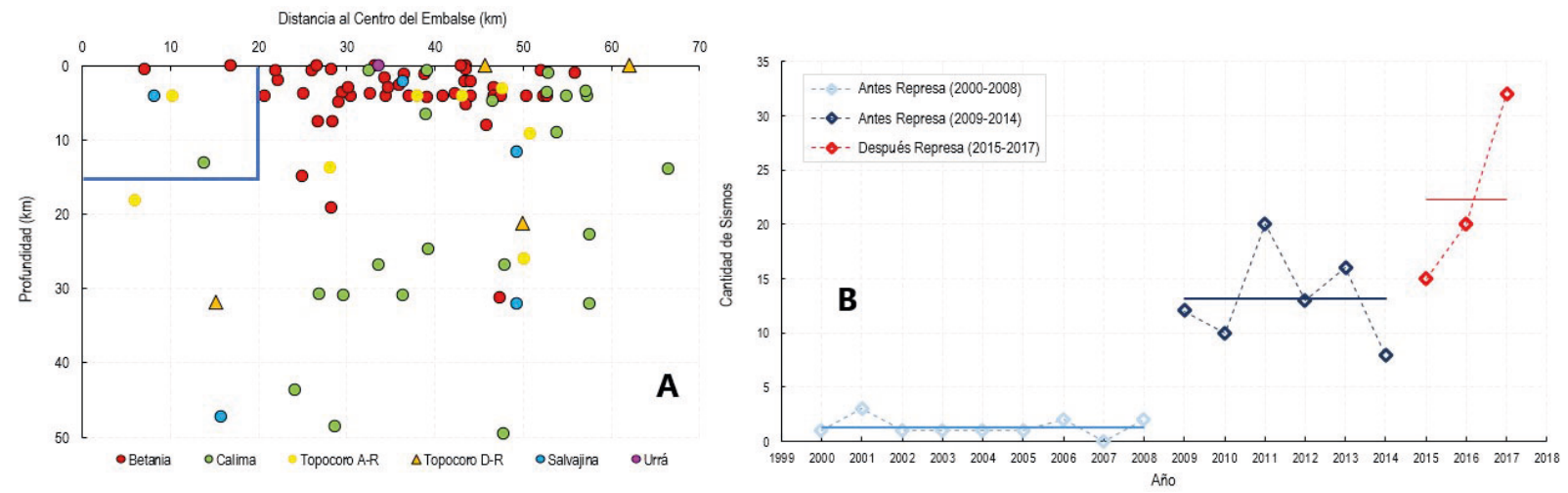

Figura 19. A. Sismos magnitud $M_{L} \geq 3,0$ registrados en cada represa. B. Incremento de eventos para la represa de Topocoro entre 0 y $5 \mathrm{~km}$ de profundidad.

Por otra parte, el registro porcentual de la totalidad de la actividad sísmica localizada en las inmediaciones de Topocoro para la ventana espacio-temporal analizada refleja que, durante el período 2015-2017 se generó el $42 \%$ del total de los eventos; período que corresponde con el 16,6\% del tiempo total analizado. 

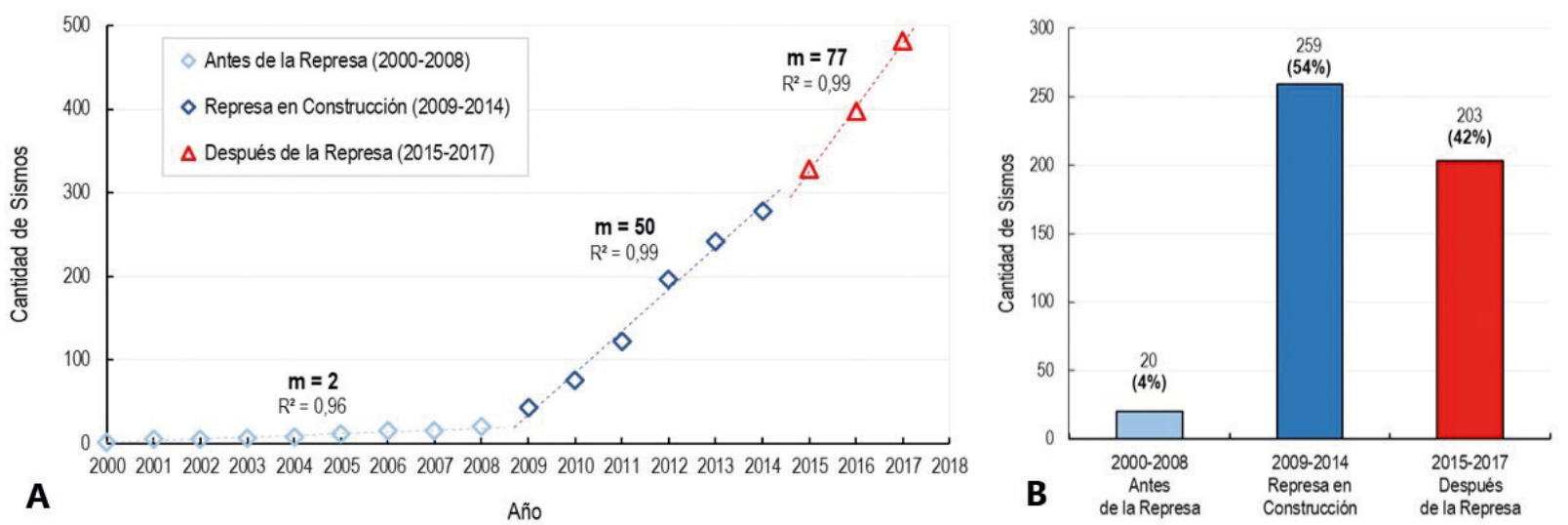

Figura 20. A. Pendiente de eventos sísmicos acumulados antes, durante y después de la construcción de la represa de Topocoro. B. Porcentaje de eventos registrados antes, durante y después de la represa.

\section{Conclusiones}

El parámetro "b" obtenido cerca del embalse de Topocoro después del ingreso en operación de la represa $(b=0,83)$ ofrece una leve tendencia a la ocurrencia de eventos de menor magnitud, en comparación con su condición inicial antes de construcción; sin embargo, el corto período de registro de datos con el cual fue determinado (2015-2017) lo hace susceptible de variación a futuro.

La teoría de Boussinesq no arrojó resultados satisfactorios para estimar la incidencia de la sobrecarga generada por los embalses frente a la ocurrencia de los eventos sísmicos, lo anterior, teniendo en cuenta la poca cantidad de eventos $M_{L} \geq 3,0$ que se correlacionaron con el bulbo de esfuerzos estimado para cada represa.

La mayor proporción de sismos $M_{L} \geq 3,0$ registrados en los alrededores de los embalses ocurrieron durante procesos de carga (o llenado) en relación con los procesos de descarga (o desembalse); localizándose a distancias usualmente mayores a los $20 \mathrm{~km}$ del centro del embalse. En complemento, al analizar la velocidad de los procesos de carga y/o descarga de los embalses, no se encontró una relación directa entre ésta y la magnitud de los eventos registrados; dado que un sismo independiente de su magnitud puede generarse en un amplio rango de velocidades.

Los análisis realizados para las represas de Betania, Calima, Urrá y Salvajina, no arrojaron una relación directa entre los eventos registrados en sus alrededores y la operación de los embalses.
Para la represa de Topocoro, los análisis realizados permiten concluir que es posible que su construcción pueda estar incidiendo en la ocurrencia de una mayor cantidad de eventos sísmicos en sus alrededores, no solamente representada en un incremento anual de eventos, sino en un aumento de los sismos registrados en los primeros $10 \mathrm{~km}$ de profundidad. Es necesario resaltar que el período de análisis de los eventos sísmicos es relativamente corto (3 años) y no permite ofrecer una afirmación contundente sobre esta relación; sin embargo, los resultados obtenidos la convierten en una represa con potencial para la generación de sismicidad antropogénica, por lo que se recomendaría más estudios empleando datos de sismicidad obtenidos con instrumentación local, que complementen la información suministrada por la RSNC para una ventana de evaluación temporal más extensa después del inicio de operación de la represa.

\section{Agradecimientos}

Los autores expresan sus agradecimientos a los profesionales Elkin de Jesús Salcedo Hurtado y Franck Albert Audemard, quienes en calidad de revisores contribuyeron con sus comentarios y sugerencias a mejorar la calidad y presentación de este trabajo.

\section{Referencias}

Barros, L.V.; Assumpção, M.; Ribotta, L.C.; Ferreira, V.M.; de Carvalho, J.M.; Bowen, B.M.; Albuquerque, D.F. (2018). Reservoir-triggered 
seismicity in Brazil: statistical characteristics in a midplate environment. Bulletin of the Seismological Society of America, 108(5B), 30463061. https://doi.org/10.1785/0120170364

Bettinelli, P.; Avouac, J.P.; Flouzat, M.; Bollinger, L.; Ramillien, G.; Rajaure, S.; Sapkota, S. (2008). Seasonal variations of seismicity and geodetic strain in the Himalaya induced by surface hydrology. Earth and Planetary Science Letters, 266(3-4), 332-344. https://doi.org/10.1016/j. epsl.2007.11.021

Consorcio Generación Ituango. (2011). Actualización estudio de impacto ambiental - Plan de contingencia. Empresas Públicas de Medellín EPM.

Dahm, T.; Hainzl, S.; Becker, D.; Bisschoff, M.; Cesca, S.; Dost, B.; Fritschen, R.; Kuhn, D.; Lasocki, S.; Klose, C.D.; Meier, C.D.; Ohrnberger, M.; Rivalta, E.; Shapiro, S.; Wegler, U. (2010). How to discriminate induced, triggered and natural seismicity. Workshop Induced Seismicity, Luxemburgo.

Das, B.M. (2012). Fundamentos de ingeniería de cimentaciones. (7ma ed.). Cengage Lerning Inc.

Drakatos, G.; Papanastassiou, D.; Papadopoulos, G.; Skafida, H.; Stavrakakis, G. (1998). Relationship between the 13 May 1995 Kozani-Grevena (NW Greece) earthquake and the Polyphyto artificial lake. Engineering Geology, 51(1), 65-74. https:// doi.org/10.1016/S0013-7952(98)00041-6

Flórez-Hurtado, J.A.; Osorio-Hoyos, L.M.; MonsalveJaramillo, H. (2013). Determinación de modelo unidimensional de velocidad en el centrooccidente colombiano utilizando tomografía sísmica local. Revista de Investigaciones Universidad del Quindío, 24(2), 245-254.

Gahalaut, V.K.; Gahalaut, K.; Catherine, J.K.; Sreejith, K.M.; Agrawal, R.; Yadav, R.K.; Mohanalaksmi, C.; Naidu, M.S.; Rajeshwar, V. (2018). Geodetic constraints on tectonic and anthropogenic deformation and seismogenesis of Koyna-Warna region, India. Bulletin of the Seismological Society of America, 108(5B), 2933-2942. https:// doi.org/10.1785/0120170373
Gómez, J.; Nivia, A.; Montes, N.E.; Almanza, M.F.; Madrid, F.A. (2015). Notas explicativas: Mapa Geológico de Colombia, Compilando la geología de Colombia: Una visión a 2015. Servicio Geológico Colombiano, Publicaciones Geológicas Especiales, 33, 9-33.

González de Vallejo, L.I.; Ferrer, M.; Ortuño, L.; Oteo, C. (2006). Ingeniería Geológica. Pearson Prentice Hall.

González de Vallejo, L.I.; Rodríguez, J.A.; Insúa, J.M.; García, J.; Mazariegos, A.; Oliveira, R.; Bommer, J. (2005). Informe de supervisión de los estudios y análisis disponibles sobre la seguridad de la presa de Itoiz. Parte I: Sismicidad en el entorno del embalse de Itoiz. Ministerio de Medio Ambiente, España.

Gupta, H.K. (2001). Short-term earthquake forecasting may be feasible at Koyna, India. Tectonophysics, 338(3-4), 353-357. https://doi.org/10.1016/ S0040-1951(01)00083-X

Gupta, H.K. (2002). A review of recent studies of triggered earthquakes by artificial water reservoirs with special emphasis on earthquakes in Koyna, India. Earth-Science Reviews, 58(3-4), 279-310. https://doi.org/10.1016/S0012-8252(02)00063-6

Gupta, H.; Purnachandra, N.; Roy, S.; Arora, K.; Tiwari, V.M.; Patro, P.K.; Satyanarayana, H.V.S.; Shashidhar, D.; Mallika, K.; Akkiraju, V.V.; Goswami, D.; Vyas, D.; Ravi, G.; Srinivas, K.N.S.S.S.; Srihari, M.; Mishra, S.; Dubey, C.P.; Raju, D.C.V.; Borah, U.; Chinna, K.; Babu, N.; Rohilla, S.; Dhar, U.; Sen, M.; Bhaskar, Y.J.; Bansal, B.K.; Nayak, S. (2015). Investigations related to scientific deep drilling to study reservoir-triggered earthquakes at Koyna, India. International Journal of Earth Sciences, 104(6), 1511-1522. https://doi.org/10.1007/s00531-014$1128-0$

Haggag, H.M.; Bhattacharya, P.M.; Kamal, S.; Kayal, J.R. (2009). Seismicity and 3D velocity structure in the Aswan Reservoir Lake area, Egypt. Tectonophysics, 476(3-4), 450-459. https://doi. org/10.1016/j.tecto.2009.07.015

Lizurek, G.; Wiszniowski, J.; Giang, N.V.; Plesiewicz, B.; Van, D.Q. (2017). Clustering and stress 
inversion in the Song Tranh 2 reservoir, Vietnam. Bulletin of the Seismological Society of America, 107(6), 2636-2648. https://doi. org/10.1785/0120170042

McGarr, A.; Simpson, D.; Seeber, L. (2002). Case histories of induced and triggered seismicity. In: W.H.K. Lee; H. Kanamori; P.C. Jennings; C. Kisslinger (eds.). International Handbook of Earthquake and Engineering Seismology (pp. 647661). Volume 81, Part A, Chapter 40. Elsevier Inc. https://doi.org/10.1016/S0074-6142(02)80243-1

Mikhailov, V.O.; Arora, K.; Ponomarev, A.V.; Srinagesh, D.; Smirnov, V.B.; Chadha, R.K. (2017). Reservoir induced seismicity in the Koyna-Warna region, India: Overview of the recent results and hypotheses. Izvestiya, Physics of the Solid Earth, 53(4), 518-529. https://doi. org/10.1134/S1069351317030041

Montalvo-Arrieta, J.C.; Pérez-Campos, X.; RamosZuñiga, L.G.; Paz-Martínez, E.G.; Salinas-Jasso, J.A.; Navarro de León, I.; Ramírez-Fernández, J.A. (2018). El Cuchillo seismic sequence of October 2013-July 2014 in the Burgos basin, northeastern Mexico: hydraulic fracturing or reservoir-induced seismicity? Bulletin of the Seismological Society of America, 108(5B), 3092-3106. https://doi. org/10.1785/0120180165

Muñoz-Burbano, F.J.; Vargas-Jiménez, C.A.; Chicangana, G. (2015). Sismicidad en el piedemonte llanero colombiano: caracterización, relocalización y tomografía sísmica local. Boletín de Ciencias de la Tierra, 38, 14-24. https://doi. org/10.15446/rbct.n38.45681

Palacios, R.A. (2013). Inventario documentado de represas en Colombia. Tesis, Universidad Militar Nueva Granada, Bogotá, Colombia.

Pérez, J.L.; Salcedo-Hurtado, E.J.; Mora-Páez, H. (2014). Análisis sismotectónico regional como contribución al estudio de las fuentes sismogénicas locales en la zona del embalse Calima, Valle del Cauca, Colombia. Boletín de Geología, 36(2), 101-124.

Simpson, D.W.; Leith, W.S.; Scholz, C.H. (1988). Two types of reservoir-induced seismicity. Bulletin of the Seismological Society of America, 78(6), 2025-2040.

Stiros, S.C.; Pytharouli, S. (2018). Interpretations of reservoir-induced seismicity may not always be valid: The case of seismicity during the impoundment of the Kremasta Dam (Greece, 1965-1966). Bulletin of the Seismological Society of America, 108(5B), 3005-3015. https://doi. org/10.1785/0120170359

Urban, P.; Lasocki, S.; Blascheck, P.; do Nascimento, A.F.; Giang, N.V.; Kwiatek, G. (2016). Violations of Gutenberg-Richter relation in anthropogenic seismicity. Pure and Applied Geophysics, 173(5), 1517-1537. https://doi.org/10.1007/s00024-0151188-5

Zhang, L.; Li, J.; Wei, G.; Liao, W.; Wang, Q.; Xiang, C. (2017). Analysis of the relationship between water level fluctuation and seismicity in the Three Gorges Reservoir (China). Geodesy and Geodynamics, 8(2), 96-102. https://doi. org/10.1016/j.geog.2017.02.004

Zhang, L.; Li, J.; Sun, X.; Liao, W.; Zhao, Y.; Wei, G.; He, C. (2018). A possible mechanism of reservoir-induced earthquakes in the Three Gorges Reservoir, central China. Bulletin of the Seismological Society of America, 108(5B), 30163028. https://doi.org/10.1785/0120180015

Trabajo recibido: mayo 04 de 2020

Trabajo aceptado: agosto 25 de 2020 